\section{The characteristics and spatial distributions of initially missed and rebiopsy-detected prostate cancers}

\author{
Myung-Won You ${ }^{1,2}$, Mi-hyun Kim¹, Jeong Kon Kim', Kyoung-Sik Cho \\ ${ }^{1}$ Department of Radiology, Research Institute of Radiology, Asan Medical Center, University \\ of Ulsan College of Medicine, Seoul; ${ }^{2}$ Department of Radiology, Eulji Medical Center, Eulji \\ University School of Medicine, Seoul, Korea
}

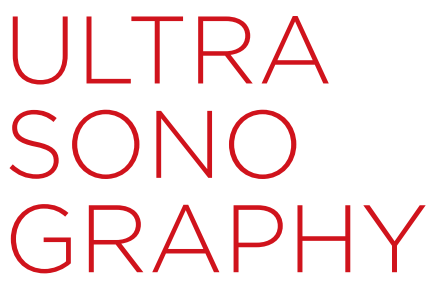

ORIGINAL ARTICLE

http://dx.doi.org/10.14366/usg. 15065 pISSN: 2288-5919 • elSSN: 2288-5943 Ultrasonography 2016:35:226-233

Received: October 11, 2015

Revised: February 4, 2016

Accepted: February 12, 2016

Correspondence to:

Mi-hyun Kim, MD, Department of Radiology, Research Institute of Radiology, Asan Medical Center, University of Ulsan College of Medicine, 88 Olympic-ro 43-gil, Songpa-gu, Seoul 05505, Korea

Tel. +82-2-3010-4386

Fax. +82-2-476-0090

E-mail:kmh0214@gmail.com

This is an Open Access article distributed under the terms of the Creative Commons Attribution NonCommercial License (http://creativecommons.org/ licenses/by-nc/3.0/) which permits unrestricted noncommercial use, distribution, and reproduction in any medium, provided the original work is properly cited.

Copyright $\odot 2016$ Korean Society of Ultrasound in Medicine (KSUM)

Keywords: Prostatic neoplasms; Image-guided biopsy; Ultrasonography

\section{Introduction}

Prostate cancer is the second most common malignancy in men, and its incidence is rapidly increasing with prostate-specific antigen (PSA)-based screening [1]. PSA is the most widely used biomarker for screening of prostate cancer, and an elevated serum PSA value leads to the performance of transrectal ultrasound (TRUS)-guided biopsy in order to identify and diagnose a prostate cancer [2].

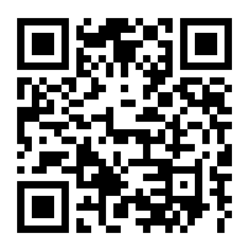

How to cite this article:

You MW, Kim MH, Kim JK, Cho KS. The characteristics and spatial distributions of initially missed and rebiopsy-detected prostate cancers. Ultrasonography. 2016 Jul;35(3):226233. 
12-Core systematic biopsy has been routinely performed under sonographic guidance as TRUS has limitations for cancer detection due to its low sensitivity and variable accuracy [3]. The reported positive predictive value of TRUS-guided prostate biopsy is $83.3 \%$, although the negative predictive value is only $36.4 \%$, indicating that negative prostate biopsy does not exclude the possibility of prostate cancer [4]. The false-negative rate of TRUS-guided prostate biopsy may be as high as $47 \%$ [5]. To reduce false-negative rates and improve detection rates of prostate cancer, radiologists should understand the characteristics of an initially missed prostate cancer, including the spatial distribution, tumor burden, and the agreement rate between the biopsy and surgical specimens.

Mazal et al. [6] described the spatial distribution of prostate cancers that were undetected on initial needle biopsy and suggested that the apico-dorsal peripheral zone should be examined on repeat biopsy. Recently, Eminaga et al. [7] analyzed the spatial distribution of prostate cancers detected on repeat biopsy and suggested that the entire anterior portion of the prostate should be inspected during the repeat biopsy. In clinical practice, most prostate cancers are diagnosed by TRUS-guided biopsy, and the importance of the concordance or discordance rate of biopsy and surgical specimens cannot be over-emphasized. In this study, we evaluated the characteristics of initially missed prostate cancer on needle biopsy based on both the biopsy and surgical specimens. Cancer positive rates, estimated tumor burden, sensitivity of core biopsy, and agreement rates of cancer positive cores between biopsy and surgical specimens were analyzed.

\section{Materials and Methods}

This retrospective study was approved by our Institutional Review Board, and the requirement for informed consent was waived.

\section{Patients}

Between January 2005 and June 2013, we identified 719 patients with negative results for cancer on initial TRUS-guided 12-core biopsy followed by a repeat biopsy performed at our medical institution. Among them, 241 patients who underwent repeat TRUSguided biopsy within 12 months after the initial negative biopsy were identified. The remaining 478 patients, who underwent repeat biopsy more than 12 months after the initial biopsy, were excluded. Indications for repeat biopsy were (1) persistently elevated PSA level $(>3.0 \mathrm{ng} / \mathrm{mL}$ ) or (2) initial atypical results of either atypical small acinar proliferation (ASAP) or prostatic intraepithelial neoplasia (PIN) [8]. We limited the interval between the previous and the repeat biopsy to "within 12 months" to exclude the possibility of de novo cancer developing more than 12 months after the previous biopsy.
Of the 241 patients, 54 were selected who demonstrated cancer on repeat biopsy. Nine patients who had not undergone prostatectomy were excluded, and 45 patients (mean age, 62.57 \pm 5.54 years; range, 45 to 78 years) were finally included. Clinical and pathologic data, including age, TRUS-measured prostate volume, surgical Gleason score, pathologic tumor stage and the number and locations of positive biopsy cores and positive compartments in the surgical specimen were recorded.

Between June 2012 and June 2013, a total of 470 patients with initially detected prostate cancer on TRUS-guided 12-core biopsy were collected. The TRUS-guided biopsy was performed following the detection of an elevated PSA level $(>3.0 \mathrm{ng} / \mathrm{mL})$. These control patients were matched 1:1 with the rebiopsy group according to PSA level $( \pm 0.5)$. After excluding 10 patients without surgical specimens, 45 patients were selected as the control group.

\section{Prostate Biopsy Technique}

Before TRUS-guided prostate biopsy, all patients provided written, informed consent. The prostate biopsy procedure was as follows: fluoroquinolone antibiotics were administered orally for 3 days before the biopsy and intravenous injection of cephalosporin antibiotics was administered just before the biopsy. A bowelcleansing enema was done on the morning of the biopsy. All patients received local anesthesia using a 22-gauge spinal needle passed through the biopsy guide channel with $10 \mathrm{~mL} 2 \%$ lidocaine injected into each neurovascular bundle. The three-dimensional diameter of the prostate was measured using a Sequoia 512 unit (Acuson, Montain View, CA, USA) or a Philips IU-22 unit (Philips Ultrasound, Bothell, WA, USA) and a 7-MHz probe. The prostate volume was then calculated according to the prolate ellipsoid formula (length $\times$ width $\times$ height $\times \pi / 6$ ). Prostate cancers were usually indistinguishable from benign prostatic hyperplasia (BPH) nodules on TRUS; however, hypoechoic focal lesions or protruding bulky masses with increased vascularity were sometimes suspected to be malignant (Fig. 1). TRUS-guided, 12-core biopsies were performed using 18-gauge biopsy needles and a spring-loaded biopsy gun, providing 17-mm-length tissue cores. An additional target biopsy was performed in 19 patients in the rebiopsy group and eight patients in the control group who had a hypoechoic focal lesion visible on TRUS images. Patients were discharged from hospital two hours after the procedure, providing there were no procedurerelated complications.

The 12-core biopsy scheme is illustrated in Fig. 2. All biopsy specimens were labeled according to the biopsy site, i.e., base, middle, or apex, lateral or medial, and right or left lobe, and submitted for pathologic analysis in separate, formalin-filled containers. 

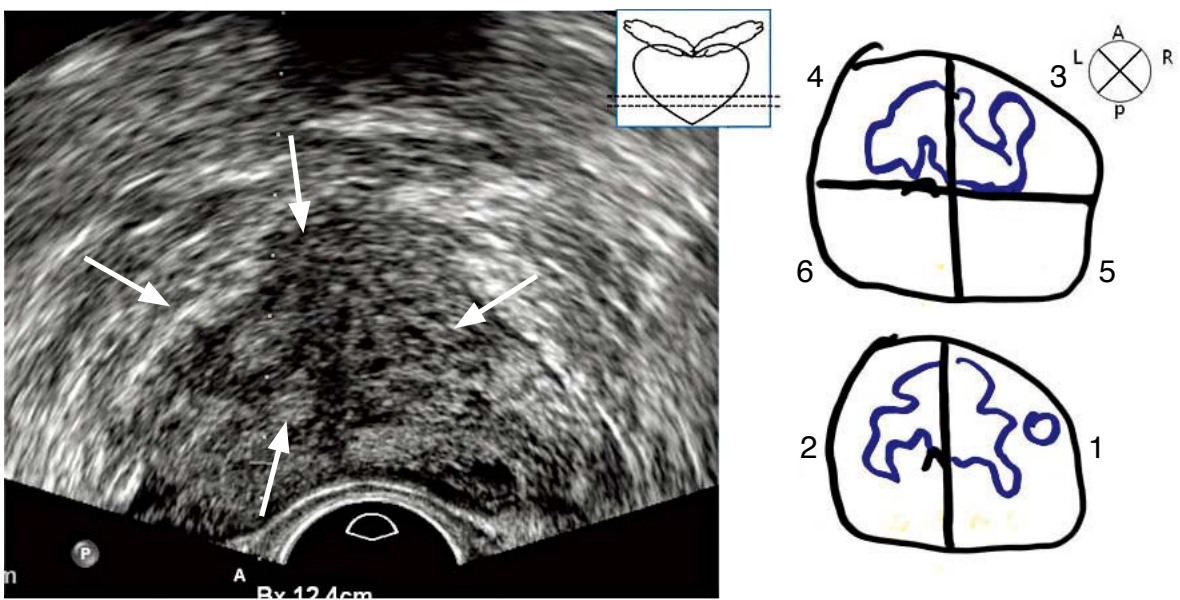

A
Fig. 1. A representative transrectal ultrasound (TRUS) image and corresponding pathology map from a 68-year-old man. A. The TRUS image reveals a cancerous mass in the apex of the central gland (arrows) as a hypoechoic focal lesion. B. The corresponding pathology map shows a bilateral tumor mass in the medial apex.

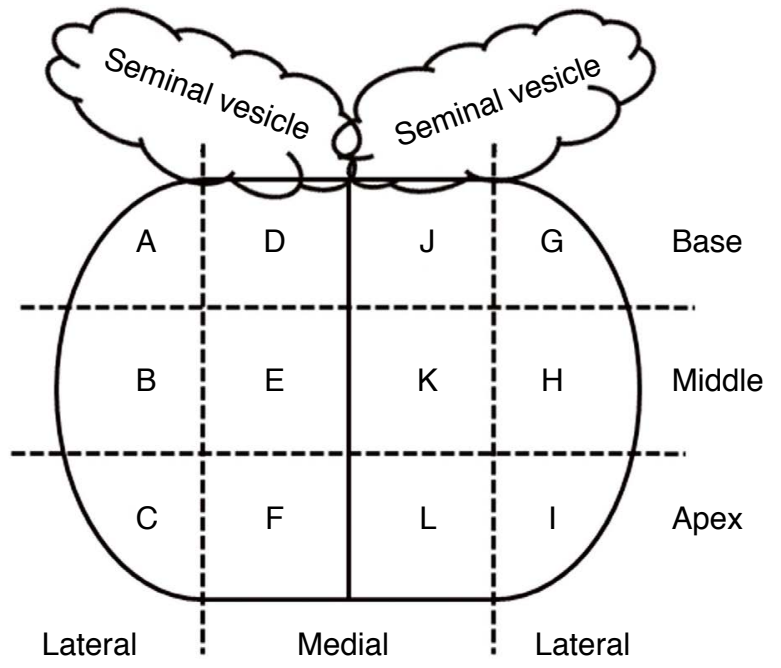

Fig. 2. Schematic diagram of 12-core needle biopsy analysis. The prostate gland is divided into 12 compartments. A, right lateral base; $B$, right lateral middle; $C$, right lateral apex; $D$, right medial base; $E$, right medial middle; $F$, right medial apex; $G$, left lateral base; $H$, left lateral middle; I, left lateral apex; J, left medial base; $K$, left medial middle; L, left medial apex.

\section{Histopathological Analysis}

Pathology assessment of the biopsy core included the length of the core, the number and location of positive cores, the percent of cancer involvement in any positive core, and the biopsy Gleason score. The histopathological analysis was based on the standard 12-core biopsy scheme as mentioned above. For radical prostatectomy specimens, several board-certified pathologists created a pathologic map of the prostate cancer in each patient after processing the surgical specimen, as described in a previous report [9]. Staff pathologists at our institution outlined the cancer foci on step-sectioned pathology slides. A prostate cancer pathology map contained drawings of 8-10 serially sectioned cut surfaces of a prostatectomy specimen from the base to the apex, and we classified the base, middle, and apex as $2 / 3 / 3,3 / 3 / 3$, or $3 / 4 / 3$ depending on the numbers of the drawn cut-surface specimens. We drew an imaginary line on the pathology map dividing lateral and medial in order to define the 12 compartments. A board-certified radiologist with 1 year of clinical experience performing TRUS-guided prostate biopsy (M.W.Y.) drew a region of interest (ROI) for each outlined cancer focus, and the estimated tumor burden was calculated as the summation of these ROls in each compartment using Photoshop (Adobe Photoshop CS5 Extended, ver. 12.0.3 x32). To calculate the tumor burden, the maximum tumor length among the overall cancer foci was measured on the pathology map. This pixel length was then converted into the logical length based on the known maximum tumor length of the prostatectomy specimen recorded in the pathology report using the set measurement menu in Photoshop. The sum of the tumor burden was calculated based on this maximum tumor length in each of the 12 compartments and represented in units of $\mathrm{cm}^{2}$ in Photoshop. Finally, the tumor burden was recorded in a unit of $\mathrm{ml}$ by multiplying slice thickness in each patient with the previously determined $\mathrm{cm}^{2}$ unit value.

We combined the right and left prostate gland, simplifying 12 compartments into six compartments; i.e., $A$, right lateral base and $G$, left lateral base as $A+G ; B$, right lateral middle and $H$, left lateral middle as $B+H ; C$, right lateral apex and I, left lateral apex as $C+l$; $D$, right medial base and J, left medial base as $D+J$; $E$, right medial middle and $\mathrm{K}$, left medial middle as $\mathrm{E}+\mathrm{K}$; and $\mathrm{F}$, right medial apex and $L$, left medial apex as $F+L$ (Fig. 2). Cancer positive rates on the biopsy and surgical specimens in each of the six compartments were then obtained in the rebiopsy and the control groups, respectively. 
The estimated tumor burden in each of the six compartments was expressed as the mean and standard deviation of the percentage and absolute tumor volumes and compared between the two groups. The agreement rate between the biopsy and surgical specimens was defined as the number of cancer positive compartments on biopsy agreeing with that on surgical specimen divided by the total number of compartments; this rate was compared between the two groups along with the sensitivity of the TRUS-guided biopsy. The cancer positive rate of target biopsy cores $(n=19)$ and ASAP and PIN lesions $(n=26)$ on rebiopsy was also evaluated.

\section{Statistical Analysis}

Since the control group was selected by 1:1 matching with the rebiopsy group, paired t tests and marginal homogeneity tests were used to compare clinical data such as age, prostate volume, number of cancer positive biopsy cores and cancer positive surgical compartments, surgical Gleason scores, and pathologic tumor stage. The cancer positive rates on biopsy cores and surgical specimen and the agreement rates of biopsy and surgical specimens were compared using a McNemar test for paired data. The estimated tumor burden was presented as mean percentage (\%) and absolute tumor volume $(\mathrm{mL})$ and the biopsy sensitivity in each compartment was compared using $t$ test or Mann-Whitney $U$ test and Fisher exact test or chi-square test, respectively. A P-value less than 0.05 was deemed significant when comparing between the two groups.

\section{Results}

\section{Comparison of Clinical Data Control vs. rebiopsy groups}

The clinical data from the control and rebiopsy groups are presented in Table 1. Patient age, surgical Gleason score and pathologic tumor stage were not different between the two groups. The prostate volume $(\mathrm{mL})$ was significantly larger in the rebiopsy group (rebiopsy, $40.79 \pm 20.71$; control, $33.14 \pm 12.21 ; P=0.032$ ). The number of cancer positive cores in the biopsy (control, 3.64 \pm 2.19 ; rebiopsy, $2.00 \pm 1.25 ; P<0.001$ ) and surgical specimens (control, 6.62 \pm 2.78 ; rebiopsy, $4.95 \pm 2.81 ; P=0.014$ ) of the control group was greater than that in the rebiopsy group.

\section{Chronological data regarding initial vs. repeat biopsy in the rebiopsy group}

The mean biopsy interval was $4.8 \pm 3.2$ months (range, 2 to 12 months), with a maximum interval of 12 months. The PSA level (initial vs. repeat biopsy, $6.42 \pm 4.51$ vs. $6.74 \pm 4.63$ ) and PSA density (initial vs. repeat biopsy, $0.18 \pm 0.15$ vs. $0.16 \pm 0.16$ ) were not changed between the two events $(P>0.05)$. Pathologic results of 45 cases in the initial needle biopsy showed ASAP ( $n=18)$, PIN ( $n=8)$, or no cancer $(n=19)$.

\section{Estimated Tumor Burden: Control vs. Rebiopsy Group}

The mean tumor volume of all six compartments was $11.38 \pm 12.24$ $\mathrm{mL}$ in the control group and $4.31 \pm 4.82 \mathrm{~mL}$ in the rebiopsy group. The control group showed a significantly larger mean tumor burden

Table 1. Comparison of clinical data in control and rebiopsy groups

\begin{tabular}{|c|c|c|c|}
\hline Characteristic & Control $(n=45)$ & Rebiopsy ( $n=45)$ & P-value \\
\hline Age (yr) & $66.69 \pm 8.66$ & $64.24 \pm 7.32$ & 0.105 \\
\hline Prostate volume (mL) & $33.14 \pm 12.21$ & $40.79 \pm 20.71$ & 0.032 \\
\hline No. of positive biopsy cores & $3.64 \pm 2.19$ & $2.00 \pm 1.25$ & $<0.001$ \\
\hline No. of positive cores in the surgical specimen & $6.62 \pm 2.78$ & $4.95 \pm 2.81$ & 0.014 \\
\hline Surgical Gleason score & & & 0.481 \\
\hline 6 & $12(26.7)$ & $15(33.3)$ & \\
\hline 7 & $25(55.6)$ & $26(57.8)$ & \\
\hline 8 & $4(8.9)$ & $3(6.7)$ & \\
\hline 9 & $4(8.9)$ & $1(2.2)$ & \\
\hline Pathologic tumor stage & & & 0.116 \\
\hline $\mathrm{T} 2 \mathrm{a}$ & $2(4.4)$ & $7(15.6)$ & \\
\hline $\mathrm{T} 2 \mathrm{~b}$ & $3(6.7)$ & $4(8.9)$ & \\
\hline $\mathrm{T} 2 \mathrm{C}$ & $27(60.0)$ & $29(64.4)$ & \\
\hline Т3а & $13(28.9)$ & $5(11.1)$ & \\
\hline
\end{tabular}

Values are presented as mean \pm SD or number (\%). 
than the rebiopsy group $(\mathrm{P}<0.001)$. The spatial distributions of cancer volume in the control and the rebiopsy groups are listed in Table 2. In the control group, the largest mean tumor burden was found in the medial middle compartment $(1.35 \pm 1.31 \mathrm{~mL})$ and the largest mean percentage tumor volume was located in the medial apex $(30.1 \pm 21.1 \%)$. In the rebiopsy group, the largest mean tumor burden and largest mean percentage tumor volume were all found in the medial apex $(44.9 \pm 29.5 \%, 0.71 \pm 0.87 \mathrm{~mL})$. Comparing the two groups, the rebiopsy group had a significantly larger mean percentage of tumor burden in the medial apex than the control group ( $44.9 \%$ vs. $30.1 \%, P=0.015)$. The mean tumor volumes in the lateral base, medial and lateral middle and lateral apex in the control group were significantly larger than those in the rebiopsy group $(\mathrm{P}<0.05)$.

Cancer Positive Rates on the Biopsy and Surgical Specimens The highest cancer positive rate on the biopsy cores was found in the medial apex in both the rebiopsy (22.2\%) and control groups (36.7\%). When comparing the two groups, the control group had significantly higher cancer positive rates in the medial and lateral middle and medial and lateral apex than the rebiopsy group $(P<0.05)$ (Supplementary Table 1).
In the rebiopsy group, the cancer positive rate of target biopsy cores was $63.1 \%$ (12/19) and the rate of ASAP or PIN was $42.3 \%$ (11/26); these rates were higher than those in all the other six compartments in both the rebiopsy and control groups.

The highest cancer positive rate on the surgical specimens was found in the medial apex in both the rebiopsy (62.2\%) and control groups $(80.0 \%)$. When comparing the two groups, the control group had a significantly higher cancer positive rate in the medial base, medial and lateral middle, and medial apex than the rebiopsy group $(\mathrm{P}<0.05)$ (Supplementary Table 2).

\section{Sensitivity of Core Biopsy and the Agreement Rate of Cancer Positive Cores between Biopsy and Prostatectomy Specimens}

The sensitivity of core biopsy and the agreement rates between the biopsy and surgical specimens in the two groups are presented in Tables 3 and 4 . The overall biopsy sensitivity of the 540 compartments (12 compartments from 45 patients) was significantly higher in the control group than in the rebiopsy group (43.4\% vs. $22.5 \%, \mathrm{P}<0.001)$. Among the six compartments, the control group showed significantly higher biopsy sensitivity than the rebiopsy group in the medial and lateral middle and the lateral apex. The

Table 2. Estimated tumor burden on surgical specimens in control and rebiopsy groups

\begin{tabular}{lcccc}
\hline \multicolumn{1}{c}{ Variable } & \multicolumn{1}{c}{ Control } & Rebiopsy & P-value \\
\hline Mean tumor volume of all six compartments $(\mathrm{mL})$ & Medial & $0.58 \pm 0.85(8.4 \pm 5.8)$ & $4.31 \pm 4.82$ & $0.32 \pm 0.26(16.3 \pm 18.5)$ \\
Base (right, left) & Lateral & $0.44 \pm 0.57(10.4 \pm 15.9)$ & $0.37 \pm 0.66(10.6 \pm 12.6)$ & $0.620(0.244)$ \\
& Medial & $1.35 \pm 1.31(26.1 \pm 14.3)$ & $0.62 \pm 0.86(29.8 \pm 25.7)$ & $0.043(0.479)$ \\
Middle (right, left) & Lateral & $1.13 \pm 0.87(28.8 \pm 17.7)$ & $0.51 \pm 0.46(34.8 \pm 29.6)$ & $0.004(0.526)$ \\
& Medial & $0.93 \pm 1.05(30.1 \pm 21.1)$ & $0.71 \pm 0.87(44.9 \pm 29.5)$ & $0.112(0.015)$ \\
Apex (right, left) & Lateral & $0.69 \pm 0.99(21.9 \pm 16.9)$ & $0.28 \pm 0.25(23.5 \pm 23.7)$ & $0.007(0.778)$ \\
\hline
\end{tabular}

Values are mean tumor volume $(\mathrm{mL}) \pm$ standard deviation in each compartment (mean percentage of tumor burden \pm standard deviation in each compartment).

Table 3. Sensitivity of biopsy in control and rebiopsy groups

\begin{tabular}{llccc}
\hline \multicolumn{1}{c}{ Variable } & & Control & Rebiopsy & P-value \\
\hline Total (540 compartments) & & $129 / 297(43.4)$ & $50 / 222(22.5)$ & $3 / 13(23.1)$ \\
Base (right, left) & Medial & $8 / 25(32.0)$ & $4 / 18(22.2)$ & 0.001 \\
& Lateral & $10 / 26(38.5)$ & $4 / 38(10.5)$ & 0.333 \\
Middle (right, left) & Medial & $24 / 56(42.9)$ & $12 / 45(26.7)$ & 0.001 \\
& Lateral & $27 / 58(46.5)$ & $19 / 56(33.9)$ & 0.039 \\
Apex (right, left) & Medial & $30 / 72(41.7)$ & $8 / 52(15.4)$ & 0.372 \\
\end{tabular}

Values are presented as number (\%).

Sensitivity=Number of cancer positive compartments on biopsy agreeing with that on surgical specimen/Number of cancer-positive compartments on the surgical specimen. 
Table 4. Agreement rate of cancer positive cores between biopsy and surgical specimens

\begin{tabular}{lcccc}
\hline \multicolumn{1}{c}{ Variable } & & Control & Rebiopsy & P-value \\
\hline Total (540 compartments) & & $340 / 540(63.0)$ & $338 / 540(62.6)$ & 0.899 \\
Base (right, left) & Medial & $61 / 90(67.8)$ & $70 / 90(77.8)$ & 0.149 \\
& Lateral & $67 / 90(74.4)$ & $65 / 90(72.2)$ & 0.732 \\
Middle (right, left) & Medial & $54 / 90(60.0)$ & $51 / 90(56.7)$ & 0.662 \\
& Lateral & $54 / 90(60.0)$ & $55 / 90(61.1)$ & 0.879 \\
Apex (right, left) & Medial & $59 / 90(65.6)$ & $45 / 90(50.0)$ & 0.035 \\
& Lateral & $45 / 90(50.0)$ & $52 / 90(57.8)$ & 0.274 \\
\hline
\end{tabular}

Values are presented as number (\%).

Agreement rate=Number of cancer positive compartments on biopsy agreeing with that on surgical specimen/Total number of compartments.

agreement rate of cancer positive cores between the biopsy and surgical specimens in the medial apex was significantly lower in the rebiopsy group than the control group ( $50.0 \%$ vs. $65.6 \%, P=0.035$ ), while the agreement rate in the other five compartments and the total of all compartments was not significantly different between the two groups.

\section{Discussion}

Our results indicate that initially missed and rebiopsy-detected prostate cancer showed significantly smaller overall tumor burden and lower sensitivity of biopsy cores. Among the six compartments measured, excluding the medial base that showed little tumor burden, the rebiopsy group showed a smaller tumor burden in four compartments (lateral base, medial and lateral middle, and lateral apex) and a larger tumor burden in the medial apex. That is, rebiopsy-detected prostate cancers tended to be more commonly distributed in the medial apex, in contrast to the relatively even tumor distribution in initially detected prostate cancers. The smaller tumor burden in the rebiopsy group probably led to the lower sensitivity of the biopsy cores. Similarly, the rebiopsy group showed lower cancer positive rates on biopsy and surgical specimens in more than half of the six compartments compared with the control group. In spite of the difference in tumor burden between the rebiopsy and the control groups, there was no difference in biochemical and histopathologic parameters, including the mean PSA level, PSA range, mean surgical Gleason score, and pathologic tumor stage. This is somewhat contrary to previous reports of a correlation between PSA level and tumor burden. Verim et al. [1] reported that the PSA level and the prostate volume were the important parameters associated with cancer detection on prostate biopsy. However, the PSA value can increase in several conditions, including BPH, prostatitis, and undetected cancers [10]. Although PSA elevation has become the most common indication for prostate biopsy, the specificity of PSA for predicting prostate cancer within the intermediate range $(4-10 \mathrm{ng} / \mathrm{mL})$, a diagnostic "grey zone" [11], is low. Among 45 patients in the rebiopsy group in our study, most had a PSA value within this "grey zone," except for four patients and similarly most had a PSA value within grey zone in the control group. In this range of PSA level, prostate volume can affect cancer detection on TRUS-guided biopsy, because BPH is also a major factor responsible for elevated PSA [12]. In our study, the total prostate volume in the rebiopsy group was significantly larger, a characteristic that might be related to the smaller tumor burden and lower sensitivity of core biopsy in this group. Although the PSA levels in the two groups were matched, the PSA density in the rebiopsy group would be therefore be lower than that in the control group.

Although the largest tumor burden in the rebiopsy group was found in the medial apex, the agreement rate of cancer positive cores between the biopsy and surgical specimens in the medial apex was significantly lower compared with the control group. Prostate cancers located in the apex can lower the cancer detection rate and are frequently the cause of false-negative results on TRUS-guided prostate biopsy $[13,14]$, as shown in our results. The prostate apex is anatomically included in the peripheral zone, and bears an increased risk of prostate cancer. However, targeting the biopsy needle to the apex was not easy due to the deep location of the apex in the lowest pelvis, especially in patients with BPH. Consequently, the apex could frequently be under-sampled when performing prostate biopsy, despite the considerable prevalence of prostate cancer.

The cancer positive rate of previous atypical results such as ASAP and PIN was $42.3 \%$ in our study, which was comparable with previous reports $[15,16]$. Precancerous lesions can be considered as under-diagnosed cancers and pose an increased risk for cancer [17]. Target biopsy of previous atypical results or abnormal findings on TRUS images represented even higher cancer positive rates, although not performed in all patients in the rebiopsy group. 
Recently, many studies have reported that image-guided target biopsy using magnetic resonance imaging (MRI) or MRI with TRUS yield a superior performance to systematic routine 12-core biopsy $[18,19]$. Therefore, additional target biopsy can be helpful in the consideration of previous atypical results, in the context of abnormal sonographic findings and when the largest tumor burden is in the medial apex.

The adequate sampling number has not yet been determined. Aganovic et al. [20] reported that a higher number of biopsy samples (mean, 14.1 vs. 10.9 with initially detected cancer) was related to a higher chance of cancer detection with repeat biopsy [20]. Since the rebiopsy group showed a larger prostate volume with a lower tumor burden, targeting more than the 12-core routine biopsy might be recommendable.

Our TRUS biopsy procedure and histopathologic analysis were based on a standard 12-biopsy scheme on both initial and repeat biopsy. We did not consider alternative sampling templates as proposed by others $[15,21]$ or obtaining separate samples of the transition zone in rebiopsy, because we were attempting to compare the cancer positive rates in biopsy cores and prostatectomy specimens and the sensitivity rate of biopsy with the same conditions between initially missed and initially detected groups. Altering the biopsy sampling template in rebiopsy could hamper comparability between the two groups and was beyond the scope of our study.

In our study, the maximum interval between initial and repeat biopsy was 12 months. There is little data regarding the criteria for differentiating de novo and missed cancers. Previous reports suggest intervals between initial and repeat biopsy of approximately 19 months $[6,22]$ or up to 3 years $[15,17]$, which are much longer than in our study.

Our study has some limitations. First, there might be some discrepancy in the correlation between 12-core prostate biopsy results and those of the pathologic map of the surgical specimen. However, this type of discrepancy is somewhat inevitable as it stems from random prostate biopsy procedure itself. Second, we calculated the tumor burden using Photoshop after drawing ROIs manually. Therefore, there could have been errors in calculating small tumor foci, which were frequently observed in the rebiopsy group, in contrast to the confluent larger tumors observed in the control group. Third, this study is retrospective, and our analysis is confined to the standardized 12-core biopsy scheme used in clinical practice at our institution and does not consider alternative biopsy templates or direct targeting of the transition zone. However, the transition zone can be included in both medial compartments, and the 12core biopsy scheme has been applied in many other centers. Further studies, including an increased number of biopsy cores or image- guided target biopsy, would be helpful additions to our study.

In conclusion, initially missed prostate cancers appear to have a smaller tumor burden with larger prostate volume and lower sensitivity rate of biopsy cores than those of initially diagnosed prostate cancers. The largest tumor burden might be located in the medial apex, with a significantly lower agreement rate between biopsy and surgical specimens in rebiopsied individuals. Target biopsy of abnormal sonographic findings or premalignant lesions such as ASAP or PIN might show higher cancer positive rates than systematic 12-core routine biopsy.

ORCID: Myung-Won You: http://orcid.org/0000-0001-6262-5784; Mi-hyun Kim: http://orcid.org/0000-0003-4623-3259; Jeong Kon Kim: http://orcid.org/0000-00015987-443X; Kyoung-Sik Cho: http://orcid.org/0000-0002-7846-5583

\section{Conflict of Interest}

No potential conflict of interest relevant to this article was reported.

\section{Acknowledgments}

This study was supported in part by the Research Fund of the Korean Society of Ultrasound in Medicine.

\section{Supplementary Material}

Supplementary Table 1. Cancer positive rates on biopsy cores in control and rebiopsy groups (http://dx.doi.org/10.14366/ usg. 15065).

Supplementary Table 2. Cancer positive rates on surgical specimens in control and rebiopsy groups (http://dx.doi.org/10.14366/ usg. 15065).

\section{References}

1. Verim L, Yildirim A, Basok EK, Peltekoglu E, Pelit ES, Zemheri E, et al. Impact of PSA and DRE on histologic findings at prostate biopsy in Turkish men over 75 years of age. Asian Pac J Cancer Prev 2013;14:6085-6088.

2. Ahn JH, Lee JZ, Chung MK, Ha HK. Nomogram for prediction of prostate cancer with serum prostate specific antigen less than 10 ng/mL. J Korean Med Sci 2014;29:338-342.

3. Halpern EJ, Strup SE. Using gray-scale and color and power Doppler sonography to detect prostatic cancer. AJR Am J Roentgenol 2000;174:623-627.

4. Salomon L, Colombel M, Patard JJ, Lefrere-Belda MA, Bellot J, Chopin D, et al. Value of ultrasound-guided systematic sextant biopsies in prostate tumor mapping. Eur Urol 1999;35:289-293.

5. Taira AV, Merrick GS, Galbreath RW, Andreini H, Taubenslag W, Curtis $R$, et al. Performance of transperineal template-guided mapping biopsy in detecting prostate cancer in the initial and 
repeat biopsy setting. Prostate Cancer Prostatic Dis 2010;13:7177.

6. Mazal PR, Haitel A, Windischberger C, Djavan B, Sedivy R, Moser E, et al. Spatial distribution of prostate cancers undetected on initial needle biopsies. Eur Urol 2001;39:662-668.

7. Eminaga 0 , Hinkelammert $R$, Abbas $M$, Titze $U$, Eltze $E$, Bettendorf $O$, et al. Prostate cancers detected on repeat prostate biopsies show spatial distributions that differ from those detected on the initial biopsies. BJU Int 2015;116:57-64.

8. Bak JB, Landas SK, Haas GP. Characterization of prostate cancer missed by sextant biopsy. Clin Prostate Cancer 2003;2:115-118.

9. Kim JK, Hong SS, Choi YJ, Park SH, Ahn H, Kim CS, et al. Wash-in rate on the basis of dynamic contrast-enhanced MRI: usefulness for prostate cancer detection and localization. J Magn Reson Imaging 2005;22:639-646.

10. Djavan B, Remzi M, Schulman CC, Marberger M, Zlotta AR. Repeat prostate biopsy: who, how and when?: a review. Eur Urol 2002;42:93-103.

11. Tang $P$, Jin $X L$, Uhlman $M$, Lin $Y R$, Deng $X R$, Wang $B$, et al. Prostate volume as an independent predictor of prostate cancer in men with PSA of 10-50 ng ml(-1). Asian J Androl 2013;15:409-412.

12. Al-Azab R, Toi A, Lockwood G, Kulkarni GS, Fleshner N. Prostate volume is strongest predictor of cancer diagnosis at transrectal ultrasound-guided prostate biopsy with prostate-specific antigen values between 2.0 and $9.0 \mathrm{ng} / \mathrm{mL}$. Urology 2007;69:103-107.

13. Sazuka T, Imamoto T, Namekawa T, Utsumi T, Yanagisawa M, Kawamura K, et al. Analysis of preoperative detection for apex prostate cancer by transrectal biopsy. Prostate Cancer 2013;2013:705865.

14. Werahera PN, Crawford ED, La Rosa FG, Torkko KC, Schulte B, Sullivan $\mathrm{HT}$, et al. Anterior tumors of the prostate: diagnosis and significance. Can J Urol 2013;20:6897-6906.

15. Zhang M, Amberson JB, Epstein Jl. Two sequential diagnoses of atypical foci suspicious for carcinoma on prostate biopsy: a followup study of 179 cases. Urology 2013;82:861-864.

16. Adamczyk P, Wolski Z, Butkiewicz R, Nussbeutel J, Drewa T. Significance of atypical small acinar proliferation and extensive high-grade prostatic intraepithelial neoplasm in clinical practice. Cent European J Urol 2014;67:136-141.

17. Chan TY, Epstein J. Follow-up of atypical prostate needle biopsies suspicious for cancer. Urology 1999;53:351-355.

18. Raskolnikov D, Rais-Bahrami S, George AK, Turkbey B, Shakir NA, Okoro $C$, et al. The role of image guided biopsy targeting in patients with atypical small acinar proliferation. J Urol 2015;193:473-478.

19. Okoro C, George AK, Siddiqui MM, Rais-Bahrami S, Walton-Diaz A, Shakir NA, et al. Magnetic resonance imaging/transrectal ultrasonography fusion prostate biopsy significantly outperforms systematic 12-core biopsy for prediction of total magnetic resonance imaging tumor volume in active surveillance patients. J Endourol 2015;29:1115-1121.

20. Aganovic D, Prcic A, Kulovac B, Hadziosmanovic O. Influence of the prostate volume, prostate specific antigen density and number of biopsy samples on prostate cancer detection. Med Arh 2012;66:4144.

21. Scattoni V, Raber M, Capitanio U, Abdollah F, Roscigno M, Angiolilli $D$, et al. The optimal rebiopsy prostatic scheme depends on patient clinical characteristics: results of a recursive partitioning analysis based on a 24-core systematic scheme. Eur Urol 2011;60:834-841.

22. Ploussard G, Nicolaiew N, Marchand C, Terry S, Allory Y, Vacherot $F$, et al. Risk of repeat biopsy and prostate cancer detection after an initial extended negative biopsy: longitudinal follow-up from a prospective trial. BJU Int 2013;111:988-996. 